\title{
Functional Ability, Physical Activity and Self-rated Health in Old Age A Cross Sectional Population-based Study in Norway
}

\author{
Lisa Forsén ${ }^{1,2, *}$, Gudrun Maria Waaler Bjørnelv ${ }^{1,4}$, Hege Bentzen ${ }^{3}$, Anne Johanne Søgaard ${ }^{1,4}$ and \\ Sidsel Graff-Iversen ${ }^{1,5}$ \\ ${ }^{1}$ Division of Epidemiology, Norwegian Institute of Public Health, Oslo \\ ${ }^{2}$ National Resource Centre for Women's health, Oslo University Hospital, Oslo \\ ${ }^{3}$ Diakonhjemmet Hospital, Oslo \\ ${ }^{4}$ Department of Health Management and Health Economics, University of Oslo, Oslo \\ ${ }^{5}$ Institute of Community Medicine, University of Tromsø, Tromsø
}

\begin{abstract}
Functional ability (FA) is here defined as having no limitations in five questions related to the ICF dimensions "Activity" and "Participation" (ICF=International Classification of Functioning). The aim was to explore how individual and contextual factors were associated with FA in each gender, and to explore to what extent physical activity (PA) and this measure of FA used in large multipurpose health surveys seem to measure different constructs, and how close the association between PA and FA was, and whether it differed within self-rated health (SRH), lifestyle and contextual factors. Methods: All men and women aged 75-77 $(\mathrm{N}=11684)$ in five counties in Norway were invited to health surveys in 20002003. 49\% participated (2626 men, 3146 women) with answers on the five questions on FA. Data were analysed using logistic regression, factor analysis, and Structural Equation Modelling (SEM). Results: Good SRH, no serious pain or stiffness in muscles and joints, interest from others, no psychological distress and no weekly use of analgesics, hypnotics, tranquilisers or antidepressants showed the highest positive associations with FA for both men and women. The SEMestimated correlation of FA and PA items was lowest in healthy elderly $(r=0.19(0.11-0.24))$ compared to those with poor health $(\mathrm{r}=0.40(0.35-0.45))$. FA and PA measured different constructs. Conclusion: According to low correlation between FA and PA in healthy men and women, we suggest that FA and PA did not measure the same construct, and impairments in functional ability did not necessarily mean low level of physical activity if SRH was good.
\end{abstract}

Keywords: International Classification of Functioning(ICF), functional ability (FA), physical activity (PA), prevalence; structural equation modelling (SEM), cross-sectional, population based, survey; self-rated health (SRH).

\section{INTRODUCTION}

Knowledge is needed about how individual factors such as gender, age, health, lifestyle and contextual factors as educational attainment, type of residence, marital status and area population density are associated with functional ability (FA) among elderly men and women. This is urgent in light of the expected demographic changes in direction of a large number of elderly combined with relatively small numbers of young and middle-aged adults in many countries [1]. The theoretical background of studies of FA is, during the recent years, often in accordance with The Disablement Process [2]. In this process, a division between individual and contextual factors is fundamental because disability can be understood as the gap between individual factors (capability) and contextual factors (demand) [2]. Similarly does International Classification of Functioning (ICF) [3] describe decrements in human function and disability as a product of a dynamic interaction between individual factors as various health

*Address correspondence to this author at the Norwegian Institute of Public Health, Po Box 4404 Nydalen, N-0403 Oslo; Tel: +4721078199;

Fax:+4721078260; E-mail: lisa.forsen@fhi.no conditions - and contextual factors divided into personal and environmental factors [4].

In ICF [3] disability is an umbrella term covering the three dimensions: Impairments, Activity limitations, and Participation restrictions. Functional ability (FA) is in the present paper defined as having no problems based on questions related to the ICF dimensions "Activity" and "Participation". We use this term FA also when referring to authors who have used other expressions for the same or nearly the same concept.

Research on determinants of FA in the elderly has progressed over the past decades [5]. However, epidemiological research utilizing data from large multipurpose health surveys where the data is not interview based, but is based on self administered questionnaires, is in an emerging phase regarding studies of FA. Validated measurement methods suitable for this type of research, is lacking. In such research the measurement methods for FA will include fewer items than the often extensive validated instruments - because the total questionnaire must not be too comprehensive. In such surveys the questions also should be easy to answer in order 
to increase the chance of getting an acceptable response rate and hopefully reliable answers.

To our knowledge, nobody has studied the associations between individual and contextual factors and FA based on few short questions in multipurpose health surveys.

Physical activity (PA) defined as "any bodily movement produced by skeletal muscles that results in energy expenditure" [6] has been identified as an important factor in maintaining FA [5]. Being able to walk and move around is by itself a part of being functionally able. Some questions measuring PA are therefore rather similar to some of the questions about FA, such as questions about the person's actual movement inside and outside the home. However, PA questions deal with what the old person actually does by use of own strength, while FA questions often deal with what people are able to do in a wide range of housing and other daily life demands and environments - with or without helping devices. When information about PA and FA is based on self reports with few questions, it can be difficult to make this distinction clear enough - and consequently PA and FA measured in this way may create an illusion that they measure the same construct.

Environments and use of helping devices for old people vary by culture [7], including socioeconomic position. However, in any culture, the associations between PA and FA in cross-sectional studies are expected to be high. But as to our knowledge nobody has compared these associations within groups of varying self-rated health (SRH), lifestyle and contextual factors.

The aim of the present study was to explore the associations between our five item measure of FA and individual and contextual factors by using data from population-based cross-sectional surveys of men and women aged 75-77 years from different regions of Norway, and to answer the following questions:

- How are individual and contextual factors associated with FA in each gender?

- To what extent do PA and five questions about FA in large multipurpose health surveys seem to measure different constructs?

- How close is the association between PA and FA, and to what extent does this association differ by self-rated health (SRH), lifestyle and contextual factors?

\section{MATERIAL AND METHODS}

\section{The Health Surveys}

In 2000 - 2003 The National Health Screening Service (part of the Norwegian Institute of Public Health since 2002) performed multi-purpose health examination surveys in five counties in Norway: Oslo [8] (the capital, urban south), Oppland, Hedmark [9] (towns and rural areas, south), Troms and Finnmark [10] (towns and rural areas, north). The surveys included questionnaires and objective measurements. Collaborators were The University of Oslo, The City of Oslo, The University of Tromsø, and municipalities in the five counties.

\section{Data Collection}

All men and women in pre specified birth cohorts were invited to participate. In this study we only included data from participants in the eldest cohort i.e. those 75-77 years old. All together 11684 were invited and 49\% participated with answers to five questions about FA. The design and procedure of the health surveys and the main questionnaire were similar in all five counties - and is described in more detail for the Oslo Health Study, web site http://www.fhi.no/ hubro-en. The main questionnaire version used for the oldest ones (75-77 years), not translated to English, contained some questions particularly tailored to older people - e.g. the five FA questions, but most questions were the same for all birth cohorts. Briefly, a letter of invitation including the main questionnaire was sent two weeks prior to the examination appointment. The examination was carried out by a team of nurses. It included measures of height, weight, and waist and hip circumferences. Body mass index (BMI, $\mathrm{kg} / \mathrm{m}^{2}$ ) and waist/hip ratio were calculated from these measures. Systolic and diastolic blood pressure (SBP and DBP) and heart rate were measured three times with an electronic devise (DINAMAP $8100 / 8101$ ). The mean of the second and third blood pressure measurement and the third heart rate count were used in analysis. Non-fasting blood samples were collected and analyzed for total cholesterol, high-density lipoprotein (HDL) cholesterol and triglycerides. All participants received a supplementary questionnaire which they were instructed to fill in at home and return by post in pre stamped envelopes. This questionnaire provided additional information on type and size of residence.

\section{Participants}

The definition of participants in the present study was people having answered all five questions about FA. This resulted in 5772 participants, 2626 men (55\% of invited) and 3146 women ( $46 \%$ of invited).

\section{Variables}

The variables used in analyses were divided into three types: outcome variables, individual factors and contextual factors. We chose variables which were of importance according to the literature on FA [5], if available from the survey. In an often cited paper describing The Disablement Process such contextual factors are referred to as extraindividual, in contrast to intra-individual factors [2]. The individual factors are closely attached to the person, such as SRH [4], PA, use of medication and body mass index (BMI). The contextual factors are extra-individual, such as educational level, marital status, type of residence and population density in the municipality.

\section{Outcome Variables}

Functional ability (FA) was measured by the five questions: Do you, because of health, have permanent limitation with respect to 1) moving around inside your residence, 2) moving outside of the residence on your own, 3) participating in organizational - and other leisure time activities, 4) using public transportation and 5) performing essential daily errands? Three alternative answers were given: no limitations, some limitations, or large limitations (Table 1). 
Table 1. Functional Ability (FA) in 75-77 Year Olds in 5 Counties in Norway based on Five Questions About How Permanent Health Problems Reduced the Respondent's Ability to do Different Activities

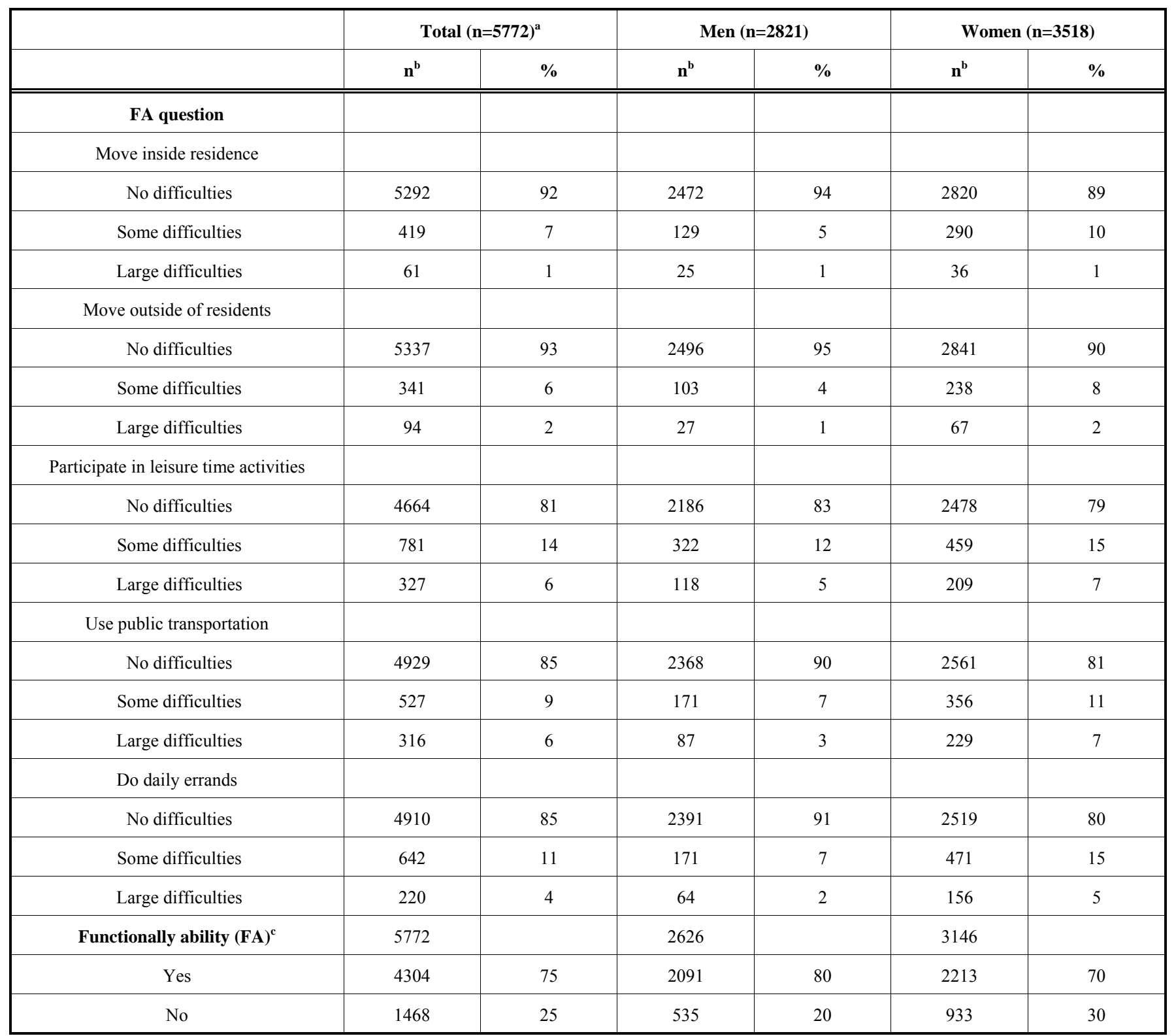

a) Total number of participants was defined as having answered all the five FA questions $n=5772$ (2626 men, 3146 women)

b) Number of participants answering the actual question

c) Having FA is defined as having answered "No difficulties" to all five FA questions

Functionally able refers to participants who answered that they had no limitations within any of these areas of FA [11].

\section{Individual Factors}

Physical activity (PA) was measured by the question "What kind of physical activity have you undertaken in the course of the past year? Estimate a weekly average for the year. The respondents were asked to mark light (making one not sweaty or out of breath) and strenuous (sweaty and out of breath) physical activity separately, and we analysed these types of PA separately, differing between none, less than 1, $1-2$, and 3 or more hours per week. "Active" was defined as all activity of one hour or more when analysing light activity, while all activity above none was defined as "active" when analysing strenuous physical activity.

BMI, $\mathrm{kg} / \mathrm{m}^{2}$ was divided into four groups: underweight (BMI $\left.<22 \mathrm{~kg} / \mathrm{m}^{2}\right)$, normal weight $\left(22-24.9 \mathrm{~kg} / \mathrm{m}^{2}\right)$, overweight $\left(\geq 25-29.9 \mathrm{~kg} / \mathrm{m}^{2}\right)$ and obese $\left(\geq 30 \mathrm{~kg} / \mathrm{m}^{2}\right)$.

Waist/hip ratio, SBP, DBP, heart rate, total and HDL cholesterol and triglycerides were assessed by comparing mean values.

Nutrition and non-alcoholic drinks were measured by several questions. We combined relevant questions and utilized "Use of vegetables" dichotomised in " $\leq 1-3$ times per week" and "more frequently" and "Potatoes" dichotomised in " $\leq$ once per month" and "more frequently", and "Soft 
drinks" dichotomised in "1 glass or more per day" and "less frequently".

Intake of vitamin supplements and cod liver oil or omega $\underline{3}$ was measured in three alternatives: No, sometimes, daily.

Smoking and alcohol: Smoking was measured by selfreports of being a never-, previous- or current daily smoker. Alcohol consumption was measured by a question on drinking frequency during the last year - with eight answering alternatives from never to 4-7 times per week. In the analysis alcohol consumption was grouped into four categories: never, seldom (none or a few times during the last year), sometimes (1-4 times per month) and more than once per week.

Psychological distress was measured using the HSCL-10, a 10 item version of Hopkins Symptom Check List (HSCL) [12] which is a self-administered instrument designed to measure psychological distress in population surveys. The average score in the HSCL-10 was calculated by dividing the total score on number of items answered (ranging between 1.00 'not at all' to 4.00 'extremely') [13]. The proportion with depression or anxiety score 1.85 or above according to the HSCL-10, was defined as cases [13].

Interest from others: The participants were asked how much concern and interest people showed in what the respondent was doing - differing between a lot, some, uncertain, little and none.

Self-rated health (SRH) was measured as in many other studies [14] as how the participants perceived their current health status, differing between poor, not so good, good and very good. This variable was dichotomized into poor or less good versus good or very good.

Use of medication was measured by self-report as how frequently the respondents during the last 4 weeks had used analgesics, hypnotics, tranquilisers or antidepressants, categorized into use of one or more of these 1) daily, 2) weekly but not daily, 3) less often and 4) no use. This variable was dichotomised in the analysis into "daily or weekly" versus "less often".

Muscles and joints: Self-reported pain or stiffness in muscles and joints during the last four weeks was reported as "no", "some" or "serious" in the following parts of the body: neck, shoulders, upper extremities, upper back, lower back, lower extremities and elsewhere. In the analysis we used a dichotomy: serious pain in at least one part of the body versus no serious pain.

Number of falls last year was self-reported and categorised into three categories: more than twice, 1-2 times, no times.

Self-reports of prevalent diseases were assessed for each of the following conditions - whether or not they had or ever had experienced: 1) asthma, 2) chronic bronchitis, 3) diabetes, 4) myocardial infarction, 5) angina pectoris, 6) stroke, 7) fibromyalgia, 8) osteoporosis, and 9) a hip fracture (yes or no). In the present paper we used the combined variables "lung disease" for a positive answer on asthma or chronic bronchitis and "cardiovascular disease" for a positive answer on one or more of myocardial infarction, angina or stroke.
Memory impairment was categorised into yes or no and was assessed by four short questions on the presence of impairment. The category "yes" included individuals who reported at least one memory impairment.

\section{Contextual Factors}

Years of education was self-reported as total number of years in school, and categorized into $\leq 7$ years, $8-10$ years and $>10$ years.

Type of residence: was self-reported as own house, farm, apartment, and other type of residence.

Size of residence was self-reported in square meters $\left(\mathrm{m}^{2}\right)$ by the participants and was in the analysis categorized into $<$ $80 \mathrm{~m}^{2}, 80-120 \mathrm{~m}^{2}$ and $>120 \mathrm{~m}^{2}$.

Live with others: Whether or not participants lived alone was assessed through questions on whether or not they lived with 1) a spouse (yes/no), and 2) with others (yes/no). Participants who answered yes to any of these questions were classified as living with others, while the other participants were classified as living alone.

Marital status was obtained from the national population register and categorized into four groups: 1) divorced or separated, 2) widow or widower, 3) never married or 4) married.

Country of birth was categorized as "western country" versus "other". But only 1 percent of the study population was born in a non-western country and this variable was not used in analysis.

Geography was categorized as north (Troms and Finnmark) or south (Oslo, Oppland and Hedmark), and when purposeful divided into North (Troms and Finnmark), South Inland (Oppland and Hedmark) and South City (Oslo).

Population density in the municipality of each participant was obtained from Statistics Norway and separated between rural areas $(<10000$ inhabitants in the municipality) suburban areas (10 000 - 19999 inhabitants) and urban areas $(\geq$ 20000 inhabitants).

\section{Statistical Analysis}

Statistical analysis was performed by SPSS / PASW (Statistical Package for the Social Sciences) software, version 17 including AMOS 17 with Structural Equation Modelling. Gamma correlation, preferable to Kendalls tau when many tied observations, was used when analysing the correlation between ordinal variables. When examining the consistency between the FA variables we used Cronbachs alpha. Differences in FA between men and women were analysed using Chi-square tests (Table 1). Logistic regression was used when exploring the association between FA and individual and contextual factors with adjustment for confounders (Table 3a and $\mathbf{3 b}$ ). Analysis of variances (ANOVA) was used to compare individual continual factors by FA. Exploratory factor analyses were performed to assess the structure of $\mathrm{PA}$ and FA. We used eigenvalue above 1 as criterion for extracting factors. Finally, Structural Equation Modelling (SEM) was used to analyse the relationship between FA and PA in sub groups within individual and contextual factors. The corresponding 95\% confidence intervals, bias corrected, 
were estimated by means of Bootstrap in AMOS choosing the maximum likelihood method and 500 Bootstrap samples.

\section{Ethics}

The participants in all the health surveys gave a written informed consent. The surveys have been approved by the Norwegian Data Inspectorate, and examined by the Regional Ethics Committee for Medical Research.

\section{RESULTS}

Relatively few participants had some- or large functional limitations (Table 1). Totally $75 \%(n=4304)$ of the participants with valid value on FA $(\mathrm{n}=5772)$ were categorized as functionally able, $80 \%$ and $70 \%$ among men and women respectively (Table 1). A minority of the participants, $2.5 \%$ of the men and $3,5 \%$ of the women, reported to have had assistance from their municipality's ambulant nursing service once or more often during the last year, while $4.2 \%$ of the men and $9.3 \%$ of the women had received household assistance (not shown in table).

In all areas of FA men had significantly fewer limitations compared to the women $(\mathrm{p}<0.001)$. The correlation (Gam$\mathrm{ma})$ between the FA measures were significant $(\mathrm{p}<0.001)$, and ranged between 0.88 and 0.97 (Table 2). Cronbachs alpha was 0.89 when we assessed the five questions in a reliability test.

In women there was a statistically significant association between FA and all the individual and contextual factors in the logistic regression analysis except use of vitamins, geography and whether they lived with someone or not (Table 3a and $\mathbf{3 b}$ ). In men we found a lack of significant association only for use of vitamins. The individual factors: good or very good SRH, no serious pain or stiffness in muscles and joints, interest from others, no psychological distress, no weekly use of analgesics, hypnotics, tranquilisers or antidepressants, no falls last year, and light physical activity showed the highest positive associations with FA for both men and women (Table 3a). Of the participants reporting poor or not so good health $51 \%$ were functionally able $(\mathrm{FA}=1)$, while this was the case in $91 \%$ of the healthy elderly. The most important contextual factors for both men and women were the socioeconomic indicators: years of education and size and type of residence (Table $3 \mathbf{b}$ ). In men also marital status and geography were important. ANOVA for continual individual factors showed significantly lower waist/ hip ratio and heart rate, and also higher HDL cholesterol values among the functionally able women and men. In men, total cholesterol level was slightly higher, and in women triglyceride levels were lower among the functionally able (Footnotes * Table 3a). Some of the items in Table $3 \mathbf{a}$ and $\mathbf{3 b}$ were not answered by all participants. Among the individual factors, smoking had the best response rate with $99 \%$, whereas the response to the question asking about interest from others was $92 \%$. Among the contextual factors size and type of residence had relatively low response rate $(73 \%$ and $80 \%$ respectively). Data were complete for the information from national registers.

An explorative factor analysis on seven variables (the five FA questions and the two PA questions) indicated a two dimensional structure (eigenvalue factor one $=3.7$, factor two $=1.8$, factor $3=0.6$ ). Our factor one included all the five FA questions, while factor two included the PA questions, suggesting that the FA and PA questions in these data probably measured different constructs. When we included $\mathrm{SRH}$, the factor analysis still suggested a two dimensional structure, and it suggested SRH in factor one.

The correlation between the constructs FA and PA was measured by SEM to be 0.38 using the total data (data not shown). This correlation varied in subgroups of self-rated health. Among the elderly reporting good or very good health, the correlation was relatively low $(\mathrm{r}=0.19)$, while among the elderly with poor or not so good health the correlation was higher $(\mathrm{r}=0.40)$. The confidence intervals of these correlations were not overlapping (Fig. 1a and 1b). No other significant interaction was found, neither within the individual nor within the contextual factors. The multiple group analysis in SEM for the two groups defined by SRH, achieved a chi-square value of 645.456 , degrees of freedom $(\mathrm{df})=26$, the Comparative Fit Index CFI $=0.95$, the TuckerLewis Coefficient TLI $=0.91$, and the RMSEA (Root Mean Square Error of Approximation) $=0.07$. CFI and TLI values close to 1 indicated a good fit. A value of about 0.08 or less for the RMSEA indicated a reasonable error of approximation.

Table 2. Gamma Correlations of Functional Ability (FA) based on Five Questions about How Permanent Health Problems Reduced The Respondent's Ability to do Different Activities ${ }^{\text {a,b }}$

\begin{tabular}{|c|c|c|c|c|c|}
\hline & $\begin{array}{l}\text { Move inside Resi- } \\
\text { dence }\end{array}$ & $\begin{array}{l}\text { Move Outside of } \\
\text { Residence }\end{array}$ & $\begin{array}{l}\text { Participate in Leisure } \\
\text { Time Activities }\end{array}$ & $\begin{array}{c}\text { Use Public Trans- } \\
\text { portation }\end{array}$ & $\begin{array}{l}\text { Do Daily } \\
\text { Errands }\end{array}$ \\
\hline Move inside Residence & 1 & & & & \\
\hline Move outside of residence & 0.97 & 1 & & & \\
\hline Participate in leisure time activities & 0.89 & 0.93 & 1 & & \\
\hline Do daily errands & 0.94 & 0.97 & 0.92 & 0.96 & 1 \\
\hline
\end{tabular}

a) Total number of participants was defined as having answered at least one of the FA questions $n=5772$

b) All gamma correlations were highly significant, and higher in men than women (data not shown in table). The gender differences varied between 0.02 and 0.05 
Table 3a. Individual Factors Associated with Functional Ability (FA) - Tested by Logistic Regression for Categorical Factors and with ANOVA for Continual Factors*

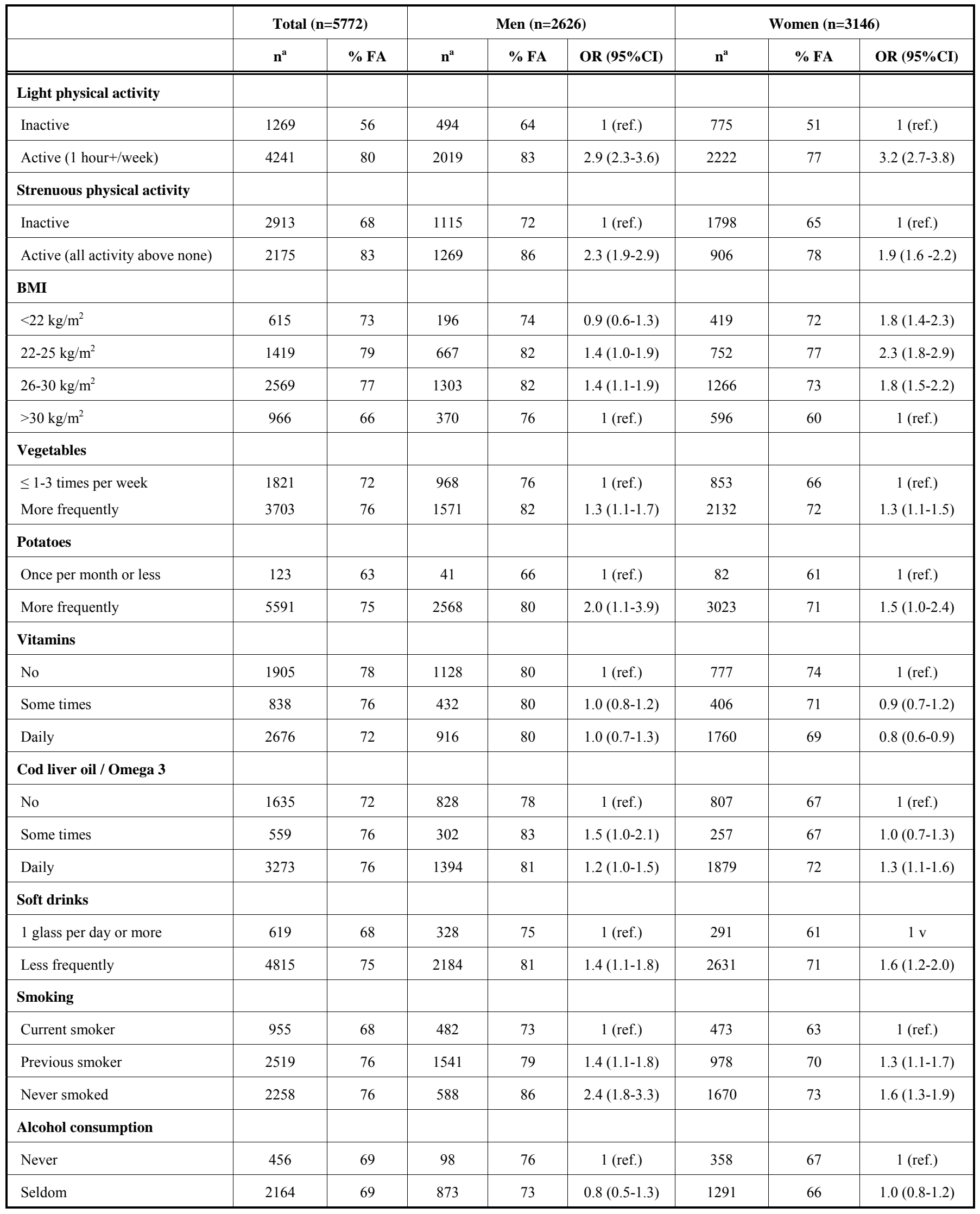


Table 3a. Contd.....

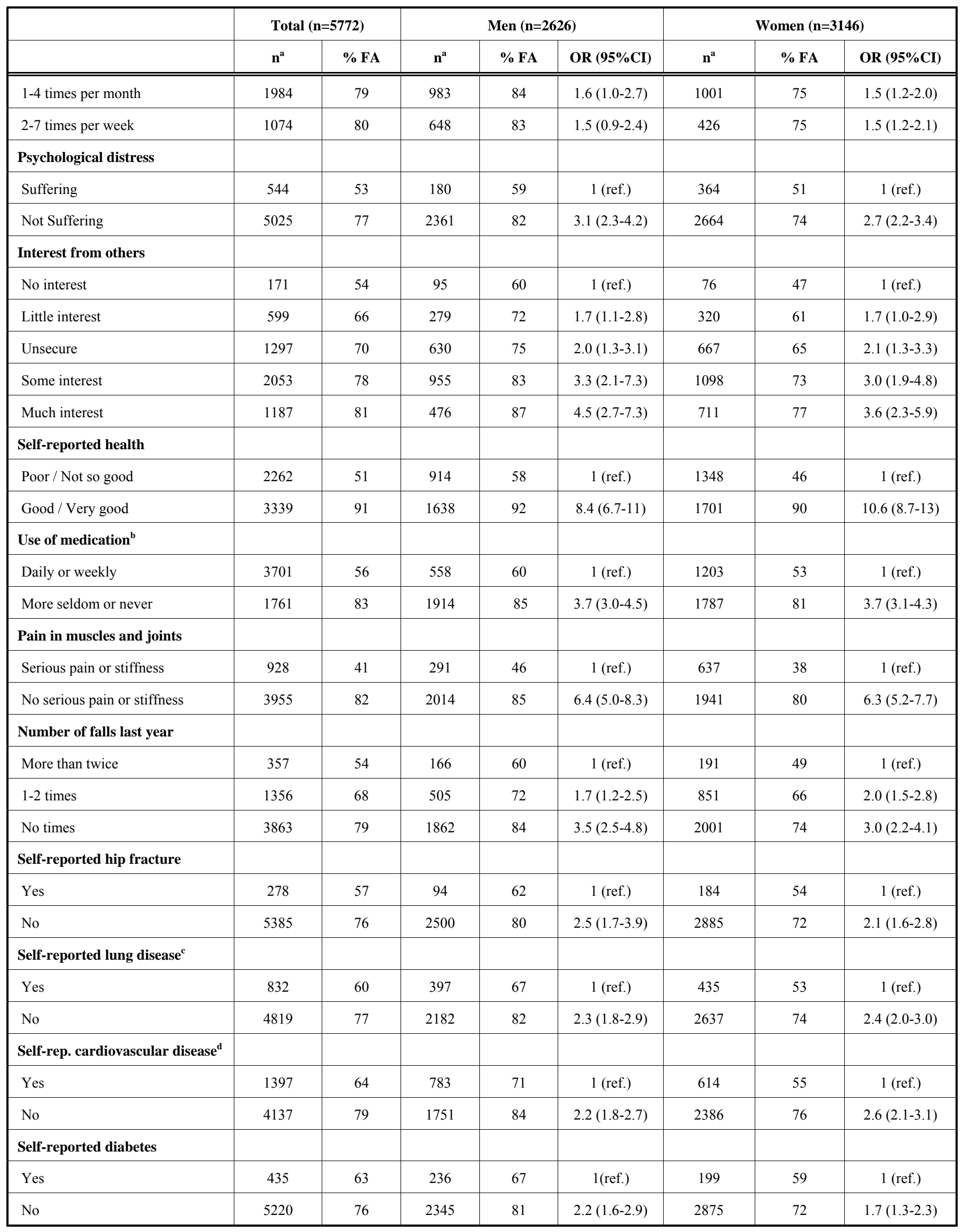


a) the number of participants answering the actual question and defined in the actual subgroup, b) use during the last 4 weeks of painkillers, sleeping pills, tranquilisers or medication for depression, c) yes, a positive answer on asthma or chronic bronchitis, d) yes, a positive answer on one or more of myocardial infarction, angina or stroke

*)

- Waist/hip ratio was significantly lower among the functionally able (both genders)

- Systolic and diastolic blood pressure did not differ significantly by FA in any of the genders

- $\quad$ Pulse frequency was significantly lower among the functionally able (both genders)

- Total and high density lipoprotein cholesterol (HDL) were significantly higher among functionally able men, and HDL cholesterol was significantly higher and triglycerides was significantly lower among the functionally able women

Table 3b. Contextual Factors Associated with Functional Ability (FA) - Tested by Logistic Regression

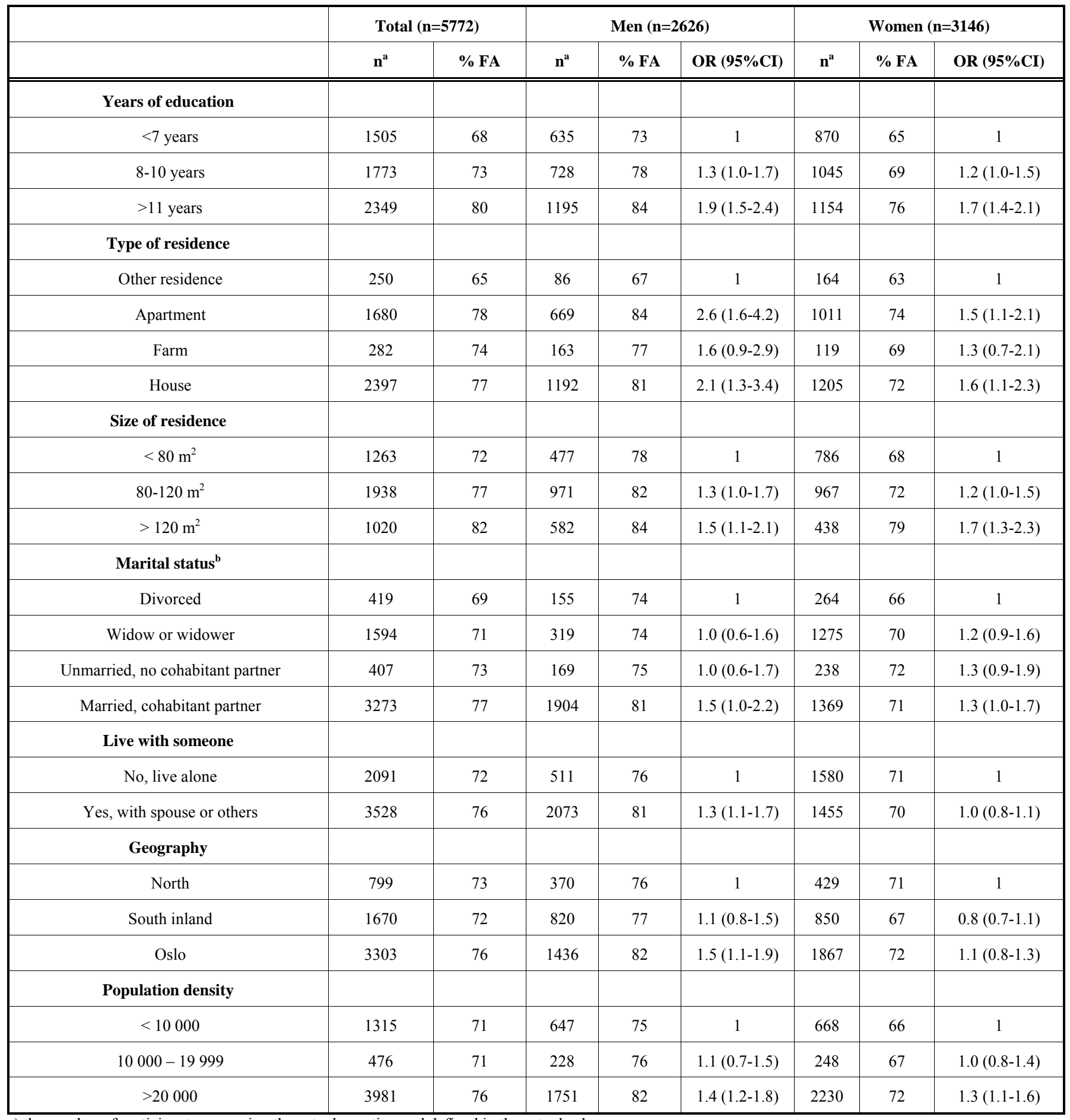

a) the number of participants answering the actual question and defined in the actual subgroup,

b) Having a cohabitant partner is in Norway an alternative to marriage 
Elderly, 75-77 year olds, with good health

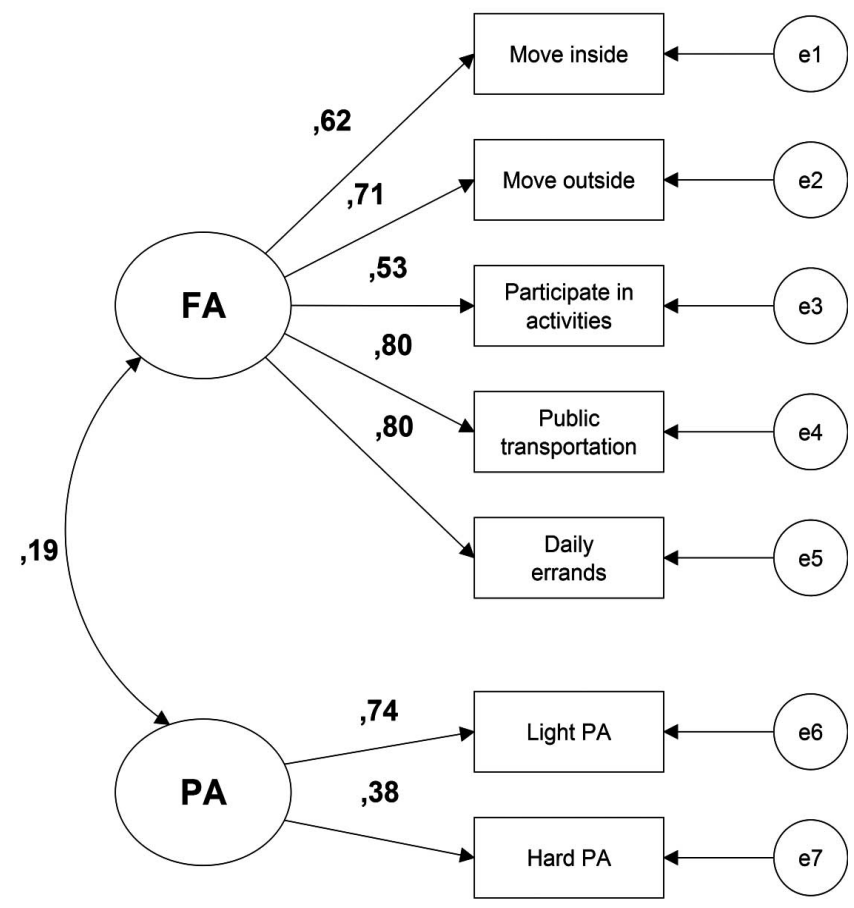

Fig. (1a). The correlation $\mathrm{r}=0.19(95 \% \mathrm{CI}, 0.09-0.25)$ between Functional ability (FA) and Physical Activity (PA) estimated by Structural Equation Modelling (SEM). N=3339. FA and PA on the figure are none-observed variables with 5 and 2 indicators respectively from the observed data, $\mathrm{e}^{1}-\mathrm{e}^{7}$ indicate none-observed measurement errors.

\section{DISCUSSION}

In this large population based cross sectional study among elderly people in Norway, the SEM correlation between Physical Activity (PA) and Functional Ability (FA) was relatively low in those reporting good health and considerably higher among those reporting less good health. The individual factors showed higher associations with FA than the contextual factors, and the strongest association was found for SRH. Also, no serious pain or stiffness in muscles and joints, much interest shown from others, no psychological distress, no weekly use of analgesics, hypnotics, tranquilisers or antidepressants, no fall last year, and weekly commitment to light physical activity were closely linked with FA. The finding that intake of potatoes and vegetables were linked with FA are in line with findings of independent walking ability being inversely associated with malnutrition [15]. Furthermore, except for the higher total serum cholesterol in functionally able men, the anthropometric measurements and serum analyses indicate a healthier metabolism [16] and higher level of physical fitness [17] among the functionally able women and men than among those with functional limitations. In contrast, functionally able men had a slightly higher mean level of total cholesterol than men with impairments, which could be due to a more adequate nutrition [15]. Men were more functionally able (80\%) than women $(70 \%)$.
Elderly, 75-77 year olds, with poor health

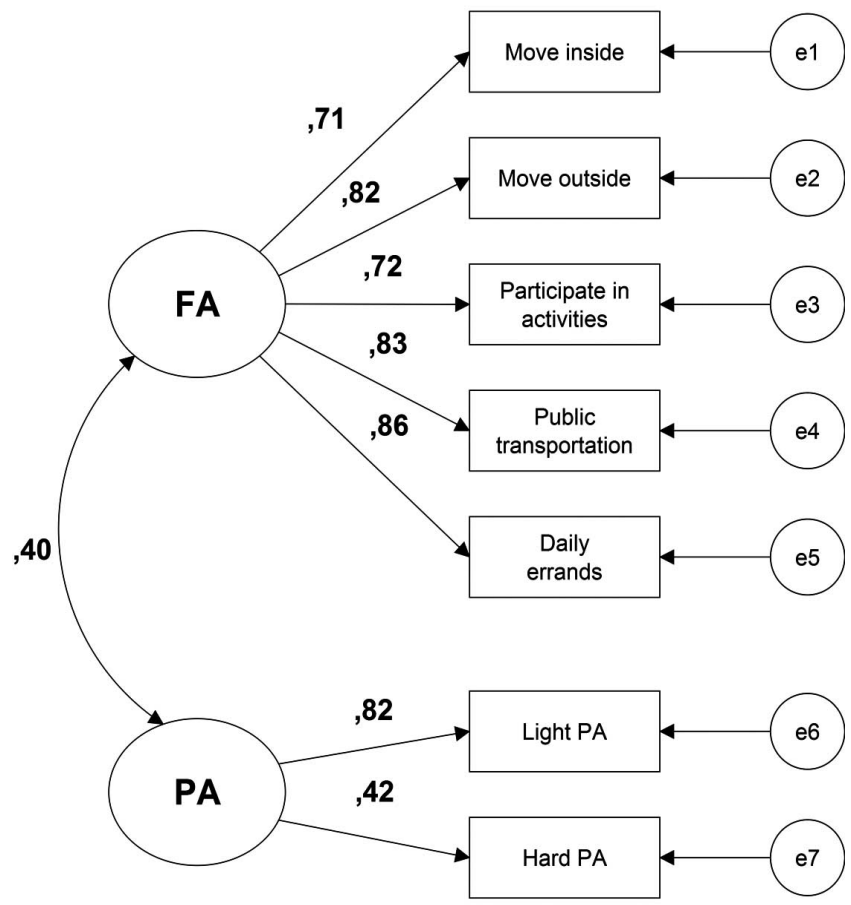

Fig. (1b). The correlation $\mathrm{r}=0.40$ (95\%CI, 0.34-0.45) between Functional Ability (FA) and Physical Activity (PA) estimated by Structural Equation Modelling (SEM). N=2262.FA and PA on the figure are none-observed variables with 5 and 2 indicators respectively from the observed data, $\mathrm{e}^{1}-\mathrm{e}^{7}$ indicate none-observed measurement errors.

The highest percentage of limitations in the five items of FA was found on the item "Participate in leisure time activities". Women had also a relatively high percentage with difficulties on "Do daily errands". These are more complex tasks and the answers may as well also reflect other difficulties than mobility limitations.

FA and PA seemed to measure different constructs in our data. SRH on the other hand, seemed to represent a construct close to FA, which is in accordance with some results in a follow-up study of survival, functional limitations and SRH [18].

Our study was population-based with 5772 participants from the capital, towns and countryside in the north, middle and south of Norway. The size was a strength of our study and made several subgroup analyses possible. However, some limitations ought to be mentioned, among them the participation rate of $49 \%$. As for many other populationbased surveys, our study is likely to be hampered by selection bias due to the relatively large number of invitees declining to participate. In the Oslo Health Study (attendance rate $46 \%$ ) - which is part of the present study, the researchers had access to information about social benefits, marital status, region of residence, education and ethnicity from all invitees. Analyses showed that the results of different health outcomes in attendees "differed only slightly from estimated prevalence values in the target population when weighted by the inverse of probability of attendance" [8]. However, for those 75/76 years old there could be an underestimation of 
prevalence of health problems in the total population when based on the participants solely. But association measures were found to be unbiased, except for ethnicity in younger individuals [8]. We had no reason to believe that the results in the counties other than Oslo - and thus the total data in our study, would be different.

Further limitations have to be mentioned: Generally, performance-based measures examine the person's ability to complete a task. In self-reports the individual to a larger degree reports the perception of his or hers ability to complete a task, and also reflects the aspect of social desirability. Thus, the associations of FA with self-reported individual factors are probably overestimated in the present study due to an unmeasured confounder, namely the individuals perception, making the measurement errors in the self-reported variables dependent [19]. Except for the measurements at the examination site and the serum analyses, all the individual factors, including FA were based on self-report. The possible overestimation of the association of FA with the individual factors would probably have been smaller if FA had been measured objectively or obtained from another resource than the survey questionnaires [19], as for example from performance-based assessments. The self-reported contextual factors were probably less influenced by this unmeasured variable and showed weaker, but still significant associations with FA. The information from national registers is not prone to this potential bias.

Our two questions about PA have not been validated in people aged 75-77, but this short PA instrument has been indirectly validated by us in the present study. Indirectly validation of variables is occasionally used in epidemiological research when a proper validation has not yet been carried out. It gives an indication that the variable behaves as expected. We compared the PA answers with results of objectively measured values of HDL-cholesterol, triglycerides and waist circumference. All the physically active groups showed significantly higher HDL-cholesterol and lower triglyserides and wais circumference than the inactive women and men. SRH is widely used both in Norway and elsewhere and has repeatedly been indirectly validated against mortality $[14,18,20,21]$. In a review of twenty-seven longitudinal community studies [14] the authors conclude that SRH represent a source of valuable data on health status. They found that SRH with five categories or less was an independent predictor of mortality in nearly all of the twenty-seven studies, despite the inclusion of numerous specific health status indicators, and other relevant covariates known to predict mortality. The authors argue that SRH represents an irreplaceable dimension of health status [14].

In the present paper all ORs in Table $\mathbf{3 a}$ and $\mathbf{3 b}$ are shown with unadjusted results. As confounding variables we have considered age, gender, years of education and marital status. The age interval was short and showed no significant association with FA, $\mathrm{p}=0.43$ and 0.41 for men and women respectively. Therefore age was not adjusted for. Gender was taken into account by separate analysis. Years of education and marital status have been adjusted for by inclusion in the models, but the results are not reported since the adjustment did not make any differences. We did not adjust for individual factors because it was uncertain whether they were con- founders or mediators or both. For example, health and musculoskeletal disorders could be confounders if poor health from younger age had limited PA, and for this reason also had reduced the FA in old age. But SRH could also be a mediator between PA and FA, by the several health promoting effects of PA.

In accordance with an earlier study [22] we found that FA among elderly Norwegian men was better than among elderly women, which might be explained by competing risk: fewer men are reaching this high age. Could a gender difference in FA explain the lower participation rate among women than among men in our study? This gender-difference in the elderly is in line with another Norwegian populationbased study [23], which found the opposite pattern in younger age groups [23]. That study also reports that poor health was the most important reason for not participating among the elderly [17]. However, a loss of FA is not the only explanation for low participation, as there are fewer car drivers among elderly women and elderly women have less often than men a spouse, which means that elderly women may have a higher threshold to come to the screening site. Further, it may be that elderly men are feeling safer than women when moving outside their homes. Fear of violence was negatively associated with physical activity for women in the Oslo Health Study [24].

In our study FA was positively associated with a number of individual factors as: light PA, low level of psychological distress, moderate consumption of alcohol, never smoking, normal body mass index, no weekly use of medication affecting the nervous system and good SRH. This harmonise well with the findings in a large review on longitudinal studies of risk factors for decreased FA in old age [5]. The review included 78 articles from 1985 to 1997 and revealed in addition that the physical context around the individual had been neglected in past research. Since then several studies have dealt with contextual variables [25-28] emphasizing that these factors can be important for maintaining FA during aging. We found an association for men, but not for women, between FA and the contextual factor "Live with spouse or others". This is in accordance with a study from Denmark [27]. Living alone significantly increased the risk of onset of disability and the risk of sustained poor FA among men, but not among single-living women - at least not in women able to participate in a survey. This may point at gender roles, with FA among elderly men being supported by a younger and healthier wife.

In both genders we found a strong positive linear association between FA and how much concern and interest people showed in what the respondent was doing. The question used was modified from one of the items in the Social Cohesion and Support Index, and has been used as one of three questions about social support in a multinational community survey [29]. In that study, the rate of depression decreased by increasing perceived interest from others [29]. This could reflect a methodological problem of dependency between variables and overestimation of correlations [19]. However, it is likely that decreased FA hampers social interactions and interest from others and subsequently leads to psychological distress. But also psychological distress could start such a vicious circle. 
Even though we did not have information about income, we had other valid indicators of socioeconomic status as years of education and size - and type of residence [30]. For "years of education" we found a positive trend for both men and women - the more years the better FA. This confirms earlier studies [31,32] which found that physical functioning increased significantly with years of education.

As to our knowledge nobody has studied the association between PA and FA in subgroups by SRH. Our study revealed that the healthiest groups had the lowest association. One reason could be that good health gives the opportunity to choose to be physically active or not, independent of having impairment in FA, while the possibilities are limited if the health is poor.

\section{CONCLUSION}

The association between Physical Activity and Functional Ability was relatively low in the healthy elderly suggesting that impairments in Functional Ability does not necessarily mean less Physical Activity when feeling that health is good in general. Thus, the challenge of maintaining Functional Ability is to maintain good health; good health encourage to Physical Activity in spite of loss of Functional Ability which again will increase the possibility of regaining Functional Ability. Male gender, good health, no serious pain or stiffness in muscles and joints, no weekly medication affecting the nervous system, much interest shown from others, no fall last year and light physical activity were the most important individual factors associated positively with Functional Ability. Also the contextual factors education and size of residence were positively associated with Functional Ability in our study, in line with a social gradient in functional health. More research on contextual factors for maintaining Functional Ability is needed in prospective studies.

\section{ACKNOWLEDGEMENTS}

We want to thank the Norwegian Institute of Public Health in delivering data from the five health surveys, the contributing research centres at the University of Tromsø and the University of Oslo, the services of all the municipalities involved and all the study participants.

The present paper was first planned and discussed in the European Network for Action on Ageing and Physical Activity (EUNAAPA) www.eunaapa.org. Thus we want to thank the European Commission, Directorate C - Public Health and Risk Assessment, for the EUNAAPA network under the Programme of community action in the field of public health (2003-2008). Further, we want to thank professor Odd Steffen Dalgard $(\dagger)$, Norwegian Institute of Public Health, Division of Mental Health, for his contribution to the discussion of the present paper.

This project has been financially supported by the Norwegian EXTRA Foundation for Health and Rehabilitation with EXTRA funds through the Norwegian Health Association.

\section{CONFLICT OF INTEREST}

The authors confirm that this article content has no conflicts of interest.

\section{REFERENCES}

[1] Christensen K, Doblhammer G, Rau R, Vaupel JW. Ageing populations: the challenges ahead. Lancet 2009; 374(9696): 1196-208.

[2] Verbrugge LM, Jette AM. The disablement process. Soc Sci Med 1994; 38(1): 1-14.

[3] World Health Organization. International classification of functioning, disability and health: ICF. Geneva: World Health Organization, World Health Organization 2001.

[4] Jette AM. Toward a common language of disablement. J Gerontol A Biol Sci Med Sci 2009; 64(11): 1165-8.

[5] Stuck AE, Walthert JM, Nikolaus T, Bula CJ, Hohmann C, Beck JC. Risk factors for functional status decline in community-living elderly people: a systematic literature review. Soc Sci Med 1999; 48(4): 445-69.

[6] Caspersen CJ, Powell KE, Christenson GM. Physical activity, exercise, and physical fitness: definitions and distinctions for health-related research. Public Health Rep 1985; 100(2): 126-31.

[7] McDowell I. Measuring health: a guide to rating scales and questionnaires. New York: Oxford University Press 2006.

[8] Søgaard AJ, Selmer RM, Bjertness E, Telle D. The Oslo health study. The impact of self selection in a large population-based survey. Int J Equity Health 2004; 3: p.3. [Online: http://www.equityhealthj.com/content $/ 3 / 1 / 3.2004]$.

[9] Hartz I, lstad I, Furu K, Skurtveit S, Elise EA. Why do sales of lipid-lowering drugs vary between counties in Norway? Evidence from the OPPHED Health Study 2000-2001. Scand J Primary Health Care 2006; 24(2): 115-21.

[10] Naess O, Sogaard AJ, Arnesen E, et al. Cohort profile: cohort of Norway (CONOR). Int J Epidemiol 2008; 37(3): 481-5.

[11] Aguero-Torres H, Thomas VS, Winblad B, Fratiglioni L. The impact of somatic and cognitive disorders on the functional status of the elderly. J Clin Epidemiol 2002;55(10): 1007-12.

[12] Derogatis LR, Lipman RS, Rickels K, Uhlenhuth EH, Covi L. The Hopkins Symptom Checklist (HSCL): a self-report symptom inventory. Behav Sci 1974;19(1):1-5.

[13] Strand BH, Dalgard OS, Tambs K, Rognerud M. Measuring the mental health status of the Norwegian population: a comparison of the instruments SCL-25, SCL-10, SCL-5 and MHI-5 (SF-36). Nordic J Psychiat 2003; 57(2): 113-8.

[14] Idler EL, Benyamini Y. Self-rated health and mortality: a review of twenty-seven community studies. [Review] [95 refs]. J Health Soc Behav 1997; 38(1): 21-37.

[15] Nykanen I, Lonnroos E, Kautiainen H, Sulkava R, Hartikainen S. Nutritional screening in a population-based cohort of communitydwelling older people. Eur J Public Health 2012 [Epub ahead of print].

[16] Grundy SM, Cleeman JI, Daniels SR, et al. Diagnosis and management of the metabolic syndrome: an American Heart Association/National Heart, Lung, and Blood Institute scientific statement. Circulation 2005; 112(17): 2735-52.

[17] Di Blasio A, Ripari P, Bucci I, et al. Walking training in postmenopause: effects on both spontaneous physical activity and training-induced body adaptations. Menopause 2012; 19(1): 23-32.

[18] Idler EL, Russell LB, Davis D. Survival, functional limitations, and self-rated health in the NHANES I Epidemiologic follow-up study, 1992. first national health and nutrition examination survey. Am J Epidemiol 2000; 152(9): 874 -83.

[19] Kristensen P. Bias from dependent errors in observational studies. [Norwegian]. Tidsskr Nor Laegeforen 2005;125(2): 173-5.

[20] Erntsen L, Nilsen SM, Espnes GA, Krokstad S. The predictive ability of self-rated health on ischaemic heart disease and all-cause mortality in elderly women and men: the Nord-Trøndelag Health Study (HUNT). Age Aging 2011; 40: 105-11.

[21] Phillips AC, Der G, Carroll D. Self-reported health, self-reported fitness, and all-cause mortality: prospective cohort study. Br J Health Psychol 2010; 15(Pt 2): 337-46.

[22] Romoren TI, Blekeseaune M. Trajectories of disability among the oldest old. J Aging Health 2003; 15(3): 548-66.

[23] Holmen J, Midthjell K, Forsen L, Skjerve K, Gorseth M, Oseland A. A health survey in Nord-Trondelag 1984-86. participation and comparison of attendants and non-attendants. Norwegian. Tid Nor Laegeforen 1990;110(15): 1973-7.

[24] Piro FN, Noss O, Claussen B. Physical activity among elderly people in a city population: the influence of neighbourhood level 
violence and self perceived safety. J Epidemiol Commun Health 2006; 60(7): 626-32.

[25] Gray DB, Hollingsworth HH, Stark S, Morgan KA. A subjective measure of environmental facilitators and barriers to participation for people with mobility limitations. Disabil Rehabil 2008; 30(6): 434-57.

[26] Lund R, Nilsson CJ, Avlund K. Can the higher risk of disability onset among older people who live alone be alleviated by strong social relations? a longitudinal study of non-disabled men and women. Age Aging 2010; 39(3): 319-26.

[27] Nilsson CJ, Lund R, Avlund K. Cohabitation status and onset of disability among older Danes: is social participation a possible mediator. J Aging Health 2008; 20(2): 235-53.

[28] Shumway-Cook A, Patla AE, Stewart A, Ferrucci L, Ciol MA, Guralnik JM. Environmental demands associated with community mobility in older adults with and without mobility disabilities. Phys Ther 2002; 82(7): 670-81.

[29] Dalgard OS, Dowrick C, Lehtinen V, et al. Negative life events, social support and gender difference in depression: a multinational community survey with data from the ODIN study. Soc Psychiatry Psychiatric Epidemiol 2006; 41(6): 444-51.

[30] Galobardes B, Lynch J, Smith GD. Measuring socioeconomic position in health research. [Review] [54 refs]. Br Med Bull 2007; 81-82: 21-37.

[31] Crimmins EM, Saito Y. Trends in healthy life expectancy in the United States, 1970-1990: gender, racial, and educational differences. Soc Sci Med 2001; 52(11): 1629-41.

[32] Ross CE, Mirowsky J. Refining the association between education and health: the effects of quantity, credential, and selectivity. Demography 1999; 36(4): 445-60.

(C) Forsén et al.; Licensee Bentham Open.

This is an open access article licensed under the terms of the Creative Commons Attribution Non-Commercial License (http://creativecommons.org/licenses/by$\mathrm{nc} / 3.0 /$ ), which permits unrestricted, non-commercial use, distribution and reproduction in any medium, provided the work is properly cited. 